\title{
AVANCES EN LA SECUENCIA CRONOLÓGICA DEL MAR INTERIOR DE CHILOÉ, PATAGONIA OCCIDENTAL: SITIOS ARQUEOLÓGICOS SAN JUAN 1, TAUCO 1 Y 2
}

\author{
OMAR REYES ${ }^{a}$, CAROLINA BELMAR ${ }^{b}$, MANUEL SAN ROMÁN ${ }^{a}$, \\ FLAVIA MORELLO ${ }^{a}$ \& XIMENA URBINA ${ }^{c}$
}

\section{RESUMEN}

En el marco de nuestras investigaciones en el archipiélago de Chiloé, se presentan nuevos datos contextuales y cronológicos de tres sitios arqueológicos registrados en la costa centro oriental de la isla de Chiloé. Los contextos descritos y su cronología reseñan ocupaciones desde el Holoceno medio y tardío, reflejando la continuidad de tradiciones cazadoras recolectoras marinas.

PALABRAS CLAVE: cazadores recolectores marinos, Holoceno medio y tardío, Chiloé, canales occidentales de Patagonia.

\section{ADVANCES IN THE CHRONOLOGICAL SEQUENCE OF CHILOÉ INTERIOR SEAS, WESTERN PATAGONIA: SAN JUAN 1, TAUCO 1 AND 2 ARCHAEOLOGICAL SITES}

\section{ABSTRACT}

As part of our research in the Chiloé archipelago, new contextual and chronological data are presented from three archaeological sites recorded on the east central coast of Chiloe Island. The described contexts and their chronology review occupations from the Middle and Late Holocene, and reflect the continuity of marine hunting and gathering traditions. Channels.

KEY WORDS: Marine Hunter-Gatherers, Middleand Late Holocene, Chiloé, Western Patagonian

\section{INTRODUCCIÓN}

La arqueología de la Isla Grande de Chiloé

ha ocupado un lugar central en la discusión arqueológica sobre el proceso de adaptación marina por parte de cazadores recolectores pescadores, su velocidad, dispersión y profundidad cronológica (Díaz \& Garretón, 1972/73; Rivas et

a Centro de Estudios del Hombre Austral, Instituto de la Patagonia, Universidad de Magallanes, Punta Arenas, Chile.

\omarreyesbaez@gmail.com, msanromanbontes@gmail.com, flavia.morello@umag.cl

b Departamento de Antropología, Universidad de Chile, Av. Capitán Ignacio Carrera Pinto 1045, Ñuñoa, Santiago, Chile. carolina_belmar@hotmail.com.

c Instituto de Historia, Pontificia Universidad Católica de Valparaíso. maria.urbina@pucv.cl 
al. 1999; Gaete et al. 2004; Ocampo \& Rivas, 2004; Orquera \& Piana, 2006; Orquera et al. 2011; San Román, 2014; Reyes et al. 2019a; Sierralta et al. 2019;), así como también las características tecnológicas de estas ocupaciones, los tipos de asentamiento y principales hitos que definen la subsistencia de estos grupos a lo largo de la secuencia temporal (Vásquez de Acuña, 1963; Stern \& Porter, 1991; Aspillaga et al. 1995; Legoupil, 2005; Munita, 2007; Álvarez et al. 2008; Rivas \& Ocampo, 2010; Álvarez et al. 2019; Urbina et al. 2020), entre otros temas de relevancia para la arqueología patagónica. En el marco de nuestras investigaciones en el archipiélago de Chiloé, damos cuenta del registro y trabajo arqueológico desarrollado en tres sitios, San Juan 1 y Tauco 1 y 2, ubicados en la costa centro oriental de Chiloé (Fig. 1), y que contribuyen con nueva información cronológica y contextual sobre la ocupación de la isla desde el Holoceno medio por parte de los grupos canoeros.

Los sitios arqueológicos San Juan 1 y Tauco 1 y 2 fueron registrados entre los años 2017 y 2018 en el marco de prospecciones dirigidas con el fin de identificar situaciones de contacto de trayectorias culturales diferentes, donde contextos formados por cazadores recolectores pescadores marinos, en momentos finales de la secuencia de ocupación del área precontacto europeo, reflejaran cambios en la subsistencia (patrón de asentamiento, tecnológicos, dieta, entre otras variables) producto de la interacción cultural con grupos de tradición hortícola procedentes del sur de Chile continental, tal cual lo reseñan algunos investigadores (Cooper, 1917, 1946; Menghin, 1962; Aldunate, 1996) y la propia data etnohistórica (Goicueta, 1558; Bibar, 1979 [1558]; Ercilla, 2009 [1569]). No obstante esta orientación temática y la consecución de estos objetivos de más largo plazo, creemos necesario entregar información cronológica y contextual obtenida hasta ahora con distinta intensidad de trabajos arqueológicos. Pretendemos con ello, dar cuenta de la diversidad de sitios descubiertos y de los elementos de diagnóstico que contribuyen a caracterizar la especialización en la subsistencia del medio litoral y marino (e.g. tecnología, materias primas, dieta), además de entregar nuevas fechas para la construcción de secuencias en la zona de estudio (e.g. Álvarez et al. 2008; Sierralta et al. 2019).

\section{MATERIALES Y MÉTODOS}

Recabando información de sitios arqueológicos conocidos por parte de la comunidad y el Museo de Castro accedimos a la localidad de Tauco y San Juan respectivamente, constatando la presencia de conchales de origen antrópico. Luego de identificados estos sitios, se procedió con el mapeo superficial y volumétrico de los depósitos, mediante la realización de barrenos, limpiezas de perfil y pozos de sondeo disponiendo de criterios estratigráficos. Las intervenciones se desarrollaron combinando el registro de niveles artificiales de 10 cm de espesor con el de la estratigrafía natural de los depósitos. Los materiales culturales, ecofactuales y bioantropológicos descubiertos fueron analizados por los diferentes especialistas.

Los análisis radiocarbónicos de los materiales recuperados fueron realizados en DirectAMS Lab. y en el Center for Applied Isotope Studies (CAIS), University of Georgia. Todas las fechas se corrigieron calibrando a $2 \sigma$ con Calib 7.0.4 (Stuiver et al. 2013) y se expresan en años antes del presente (cal AP). Para las muestras de materiales pertenecientes a cadenas tróficas terrestres $(\mathrm{N}=9$ carbón, N=5 sitio San Juan 1 y N=4 sitio Tauco 1) se aplicó la curva ShCal13 (Hogg et al. 2013). Para las muestras de individuos con dieta marina ( $\mathrm{N}=5$ óseo humano, $\mathrm{N}=4$ sitio Tauco 1 y $\mathrm{N}=1$ sitio Tauco 2) se aplicó un efecto reservorio local de $141 \pm 43$ años de acuerdo a los valores disponibles más cercanos (Merino et al. 2019). Se consideró la curva Mixed Marine SoHem (Reimer et al. 2013). La calibración contempló el porcentaje de recursos marinos en la dieta de cada uno de los individuos (Reyes et al. 2019b). Dicho cálculo se estimó a partir del $\delta^{13} \mathrm{C}$ de los individuos y de establecer dietas estimadas $100 \%$ terrestres y marinas (Coltrain et al. 2016), las cuales se determinaron en base a la ecología isotópica recopilada para el área (Reyes et al. 2019b, 2019c). Finalmente, un fragmento cerámico procedente del sitio San Juan 1 fue datado por termoluminiscencia en el Laboratorio de Dosimetría del Instituto de Física de la Universidad Católica, mediante el registro de un dosímetro enterrado para tal efecto. 


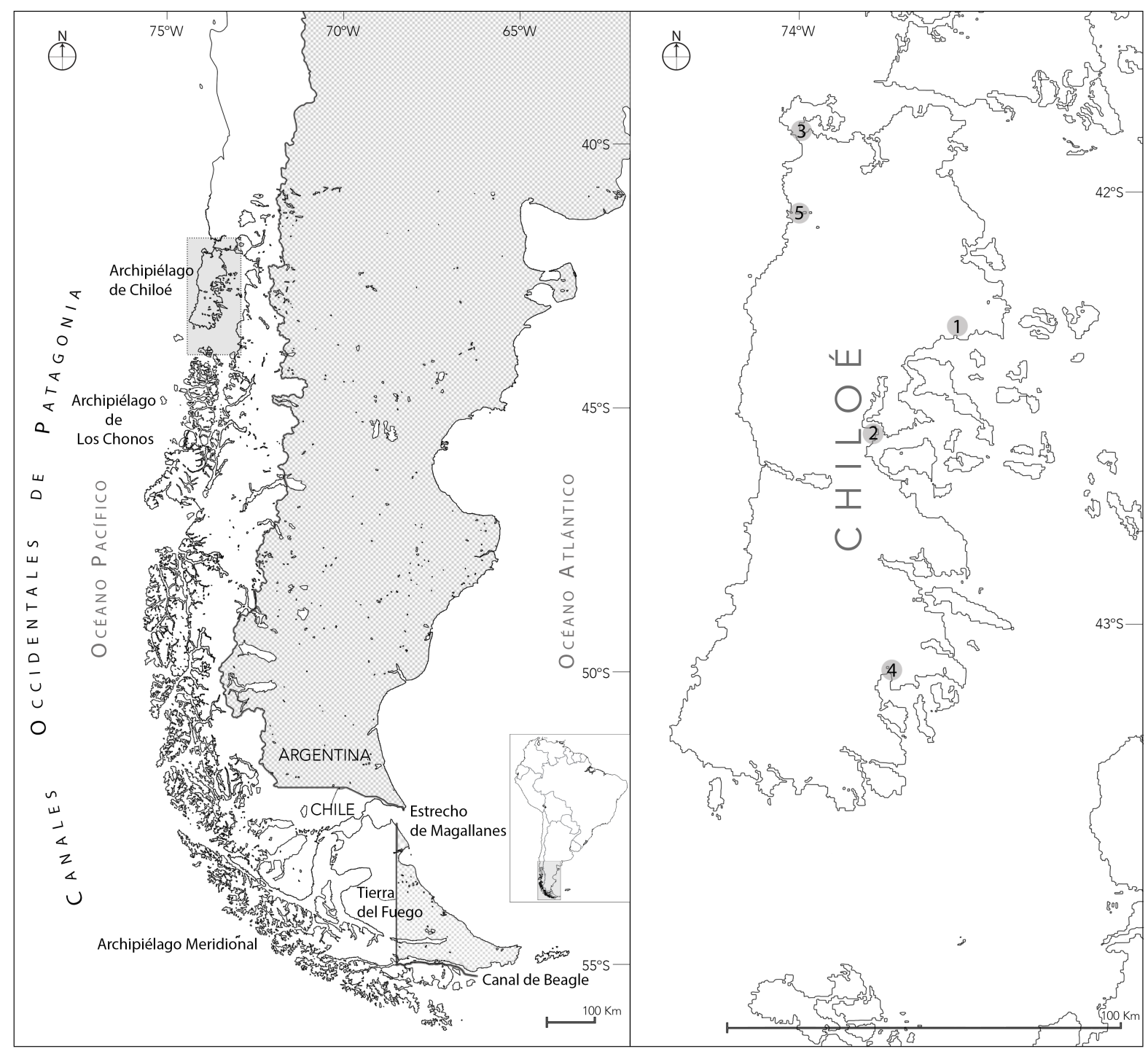

Fig. 1. Ubicación de Chiloé en Patagonia occidental y sitios mencionados en el texto.

(1) San Juan 1, (2) Tauco 1, Tauco 2 y Rauco (3), Puente Quilo 1, (4) Yaldad 2 y (5) Chepu 005.

\section{SITIO SAN JUAN 1}

Corresponde a un gran conchal ubicado bajo gran parte del pueblo de San Juan, comuna de Dalcahue (Fig. 2), en un entorno de bosque valdiviano o latifoliado siempreverde (Luebert \& Pliscoff, 2006) hoy en día muy antropizado. Éste cubre una extensa área donde es posible observarlo en chacras con tierra removida, cortes de obras y perfiles expuestos. La costa sobre la que se emplaza el sitio se orienta sur-suroeste, sobre la paleoplaya de arena formada por la planicie litoral adyacente, y la desembocadura del río San Juan en dirección norte-sur. Este río flanquea por el poniente el pueblo homónimo y el sitio arqueológico. Destacamos que en esta zona la amplitud de mareas alcanza los 6 metros (SHOA, 2019), con una zona intermareal que se proyecta centenares de metros desde la línea de altas mareas.

El conchal arqueológico se encuentra entre los 1 y 3 metros sobre el nivel actual del mar. Esta variación radica en su gran extensión. Asimismo, la distancia de la actual línea de costa varía entre los 20 y 700 m. El agua dulce del río San Juan se 


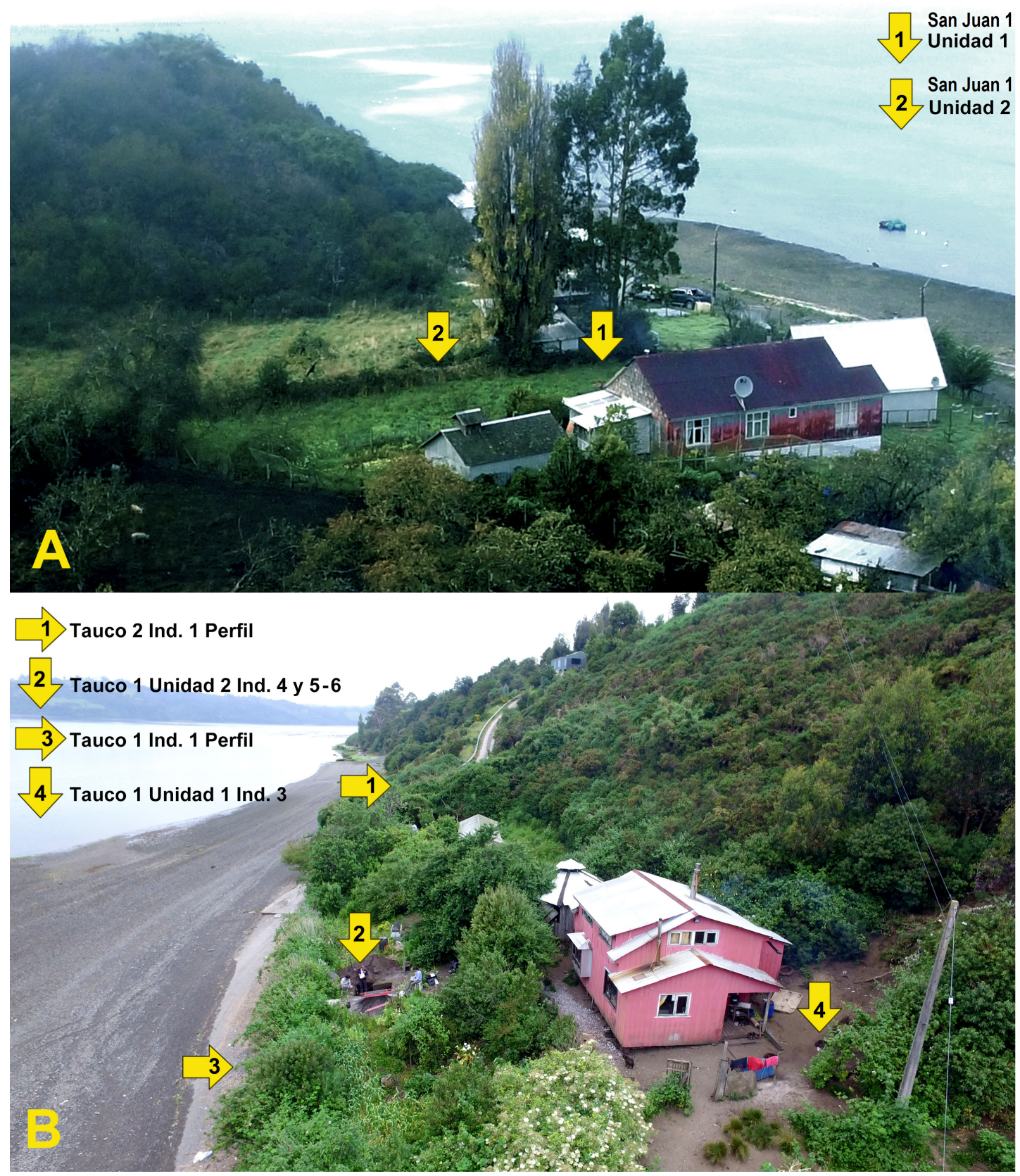

Fig. 2. Fotografías aéreas de los sitios arqueológicos e intervenciones realizadas. A. Sitio San Juan 1 y ubicación de unidades de excavación. B. Sitios Tauco 1 y 2 y ubicación de individuos en relación a las intervenciones realizadas.

encuentra actualmente a $100 \mathrm{~m}$ en marea baja. En marea alta el mar ingresa unos $1.200 \mathrm{~m}$ río arriba, siendo su acceso más distante. De acuerdo a la revisión de perfiles y los barrenados practicados, se estima que el conchal mide $170 \mathrm{~m}$ en dirección norte-sur y 100 m en dirección este-oeste cubriendo
$17.000 \mathrm{~m}^{2}$ (1,7 ha). En los barrenados, en tanto, se determinaron profundidades promedios de $\sim 230 \mathrm{~cm}$.

Se planificaron dos unidades de excavación (Fig. 2). El porcentaje de intervención corresponde al 0,020\% de la superficie estimada del yacimiento. 
La primera unidad, de $1 \times 1,5 \mathrm{~m}$, fue trazada en el borde sur del conchal, donde comienza la pendiente del mismo, hacia su borde costero. Se excavó hasta los $150 \mathrm{~cm}$ de profundidad registrando la paleoplaya. La segunda unidad, de $2 \times 1 \mathrm{~m}$, se trazó $14 \mathrm{~m}$ hacia el noreste de la primera, en la cima del conchal, en su parte más alta. Se excavó hasta los $240 \mathrm{~cm}$ de profundidad alcanzando la paleoplaya.

\section{SITIOS TAUCO 1 Y 2}

Tauco 1 es un gran conchal ubicado en la costa de la localidad de Tauco, comuna de Chonchi, situado en la costa norte del estero de Castro hacia la salida del canal Lemuy (a $25 \mathrm{~km}$ en línea recta, o $40 \mathrm{~km}$ costeando del sitio San Juan 1), a $1 \mathrm{~km}$ del conchal de Rauco con $\sim 5.000$ años cal AP (Álvarez et al. 2008), y en el mismo ambiente de bosque siempreverde antropizado (Fig. 2). Este sitio presenta un extenso perfil expuesto por la erosión de las mareas. La costa sobre la que se ubica se orienta en dirección norte, emplazándose sobre la paleoplaya de arena formada por la planicie litoral adyacente. Existe un pequeño arroyo permanente hacia el costado oeste del sitio. Los cerros que caen al mar limitan el sitio hacia su costado sur.

El conchal arqueológico se encuentra a 2,5 $\mathrm{m}$ sobre el nivel actual del mar y entre 1 y $20 \mathrm{~m}$ de la actual línea de costa en relación a la variación diaria de las mareas. De acuerdo a la revisión de perfiles y los barrenados practicados, se estima que el conchal mide $40 \mathrm{~m}$ en dirección este-oeste y 10 $m$ en dirección norte-sur, circunscrito entre la costa y el cerro que cae a su espalda, cubriendo 400 $\mathrm{m}^{2}$. En los barrenados, en tanto, se determinaron profundidades promedios de $\sim 230 \mathrm{~cm}$. Sobre este sitio se encuentra la casa, galpones y chacras, propiedad de don Francisco Aude.

El conchal Tauco 2 se ubica en el mismo emplazamiento, $30 \mathrm{~m}$ hacia el este de Tauco 1 (Fig. 2). Se trata de un pequeño conchal erosionado, visible por la exposición de su perfil norte producto de las mareas. Se encuentra a $1 \mathrm{~m}$ de la actual línea de costa y a $1 \mathrm{~m}$ sobre el actual nivel del mar. Se observa una extensión de $5 \mathrm{~m}$ en dirección esteoeste, mientras que la extensión original norte-sur, entre la costa y el cerro, no se pudo determinar con precisión debido al derrumbe de parte del cerro, siendo visible en el corte $10 \mathrm{~cm}$ de conchal. El espesor promedio estimado luego de la limpieza de perfil es de $30 \mathrm{~cm}$, correspondiendo a un locus extendido del conchal más grande Tauco 1. Es posible aseverar, no obstante, que constituye un área residual de conchal, pues el resto ha sido destruido por la acción de las mareas.

En ambos sitios el habitante del lugar, don Francisco Aude, encontró restos óseos desprendidos desde el perfil expuesto por el mar, los que identificamos como humanos, además en la playa adyacente, recuperó puntas lanceoladas de sección espesa y lascas de obsidiana riolítica. Ello nos llevó a planificar tanto la recuperación de las osamentas expuestas como la caracterización estratigráfica del lugar. En Tauco 1 se ejecutó la limpieza de perfil para rescatar los restos óseos humanos, una unidad de $1 \times 1 \mathrm{~m}$ hacia el inicio del cerro y otra de 2 x $1 \mathrm{~m}$ en el centro del conchal. El porcentaje de intervención corresponde al $0,75 \%$ de la superficie estimada del yacimiento. Se excavó hasta los $70 \mathrm{~cm}$ y $240 \mathrm{~cm}$ de profundidad alcanzando los niveles de coluvio de cerro y la paleoplaya respectivamente. En Tauco 2 en tanto se efectuó la limpieza de perfil y el rescate de las osamentas que allí se encontraban.

\section{RESULTADOS}

\section{Caracterización general de los sitios}

Sobre la cronología de ocupación del sitio San Juan 1, podemos comentar que las primeras ocupaciones que se asientan sobre la paleoplaya, en ambas unidades excavadas, se remontan al Holoceno medio, hacia $~ 6.000$ años cal AP, y continúan sucediéndose a lo largo del Holoceno tardío (Tabla 1), durante el segundo milenio y hasta el Período Alfarero Tardío, de acuerdo a la identificación de los fragmentos cerámicos monocromos recuperados $(\mathrm{N}=19)$ (Palma \& Alfaro, 2019), y tal como lo revelan los sucesivos niveles de ocupación estratigráficos. En los niveles superiores del depósito hasta los $50 \mathrm{~cm}$ promedio de profundidad, luego del contacto europeo, se registra una importante disturbación antrópica de este extenso yacimiento, principalmente por la actividad agrícola y el crecimiento del pueblo, tal como lo demuestra el material subactual registrado 
Tabla 1. Edades radiocarbónicas de los sitios San Juan 1, Tauco 1 y Tauco 2.

(-) No informado. *Fechas en nivel de cierre y base del depósito.

\begin{tabular}{|c|c|c|c|c|c|c|c|c|}
\hline Sitio & $\begin{array}{c}\text { Código } \\
\text { Laboratorio }\end{array}$ & Muestra & Material & Unidad & $\begin{array}{l}\text { Nivel } \\
\text { (en cm) }\end{array}$ & $\begin{array}{l}\text { Edad }{ }^{14} \mathrm{C} \\
\text { (años } \mathrm{AP} \text { ) }\end{array}$ & $\delta^{13} \mathrm{C}(\% \circ)$ & $\begin{array}{c}2 \sigma \mathrm{cal} \\
\text { (años AP) }\end{array}$ \\
\hline \multirow{6}{*}{ San Juan 1} & $\begin{array}{l}\text { D-AMS } \\
026734\end{array}$ & SJU1N5 & Carbón & 1 & $40-50$ & Moderno & $(-)$ & $(-)$ \\
\hline & $\begin{array}{l}\text { D-AMS } \\
026735\end{array}$ & SJU1N12 & Carbón & 1 & $110-120^{*}$ & $5164 \pm 29$ & $(-)$ & 5936-5748 \\
\hline & UCTL 3239 & $(-)$ & Cerámica & 2 & $30-40$ & $475 \pm 50$ & $(-)$ & $(-)$ \\
\hline & $\begin{array}{l}\text { D-AMS } \\
026736\end{array}$ & SJU2N8 & Carbón & 2 & $70-80$ & $2076 \pm 26$ & $(-)$ & 2080-1924 \\
\hline & $\begin{array}{l}\text { D-AMS } \\
026737\end{array}$ & SJU2N18 & Carbón & 2 & $170-180$ & $5120 \pm 27$ & $(-)$ & 5909-5743 \\
\hline & $\begin{array}{c}\text { D-AMS } \\
026738\end{array}$ & SJU2N22 & Carbón & 2 & $210-220^{*}$ & $5070 \pm 30$ & $(-)$ & 5893-5662 \\
\hline \multirow{8}{*}{ Tauco 1} & $\begin{array}{c}\text { UGAMS } \\
39582\end{array}$ & TAU1-3 & Ind.3 & 1 & $0-20^{*}$ & $3310 \pm 25$ & -11.54 & $3192-2916$ \\
\hline & $\begin{array}{l}\text { UGAMS } \\
38381\end{array}$ & TAU1-1 & Ind.1 & Perfil & $(-)$ & $2180 \pm 20$ & -11.51 & $1809-1574$ \\
\hline & $\begin{array}{c}\text { D-AMS } \\
031812\end{array}$ & TAUN9 & Carbón & 2 & $80-90$ & $2160 \pm 29$ & $(-)$ & $2298-2008$ \\
\hline & $\begin{array}{l}\text { UGAMS } \\
39583\end{array}$ & TAU1-4 & Ind.4 & 2 & $120-130$ & $2480 \pm 20$ & -11.63 & $2165-1923$ \\
\hline & $\begin{array}{l}\text { UGAMS } \\
39584\end{array}$ & TAU1-5 & Ind.5 & 2 & $120-140$ & $2410 \pm 20$ & -12.27 & 2104-1889 \\
\hline & $\begin{array}{c}\text { D-AMS } \\
031813\end{array}$ & TAUN14 & Carbón & 2 & $130-140$ & $2078 \pm 31$ & $(-)$ & 2086-1921 \\
\hline & $\begin{array}{c}\text { D-AMS } \\
031814\end{array}$ & TAUN18 & Carbón & 2 & $170-180$ & $1885 \pm 26$ & $(-)$ & $1835-1711$ \\
\hline & $\begin{array}{l}\text { D-AMS } \\
031815\end{array}$ & TAUN21 & Carbón & 2 & $200-210^{*}$ & $2156 \pm 29$ & $(-)$ & 2296-2007 \\
\hline Tauco 2 & $\begin{array}{c}\text { UGAMS } \\
39585\end{array}$ & TAU2-1 & Ind.1 & Perfil & $*$ & $2670 \pm 20$ & -11.53 & 2356-2146 \\
\hline
\end{tabular}

y la datación moderna obtenida de un trozo de carbón de aquellos niveles.

$\mathrm{El}$ análisis del material lítico recuperado ( $\mathrm{N}=1.888$, 95\% lascas y desechos de talla) señala una tecnología coherente con actividades de caza, pesca y campamento. La tecnología lítica está dominada por restos de façonnage bifacial y algunos artefactos de desbaste (Morello \& Arecheta, 2019). Solo están presentes unos pocos instrumentos, con una presencia dominante de puntas de proyectil y artefactos bifaciales $(\mathrm{N}=16)$, destacando la formatización bifacial como principal actividad de fabricación de instrumentos in situ (Fig. 3). También se registraron algunos instrumentos de retoque marginal como lascas retocadas, una raedera y un cuchillo. La tendencia dominante de la cadena operativa de manufactura de puntas de proyectil sobre obsidiana riolítica se ve reforzada por la alta frecuencia de lascas angulares e internas con un $85 \%$ de uso de percusión directa con percutor blando como técnica de aplicación de la fuerza de extracción. Otros artefactos líticos incluyen percutores, yunques y algunas piedras de molienda, entre otros. Se fabricaron principalmente mediante el uso de cantos rodados planos que muestran claras marcas de actividad como piqueteado, abrasión, pulidos, etc. Entre éstos, hay 5 instrumentos de conformación similar, pero de función indeterminada. Se trata 

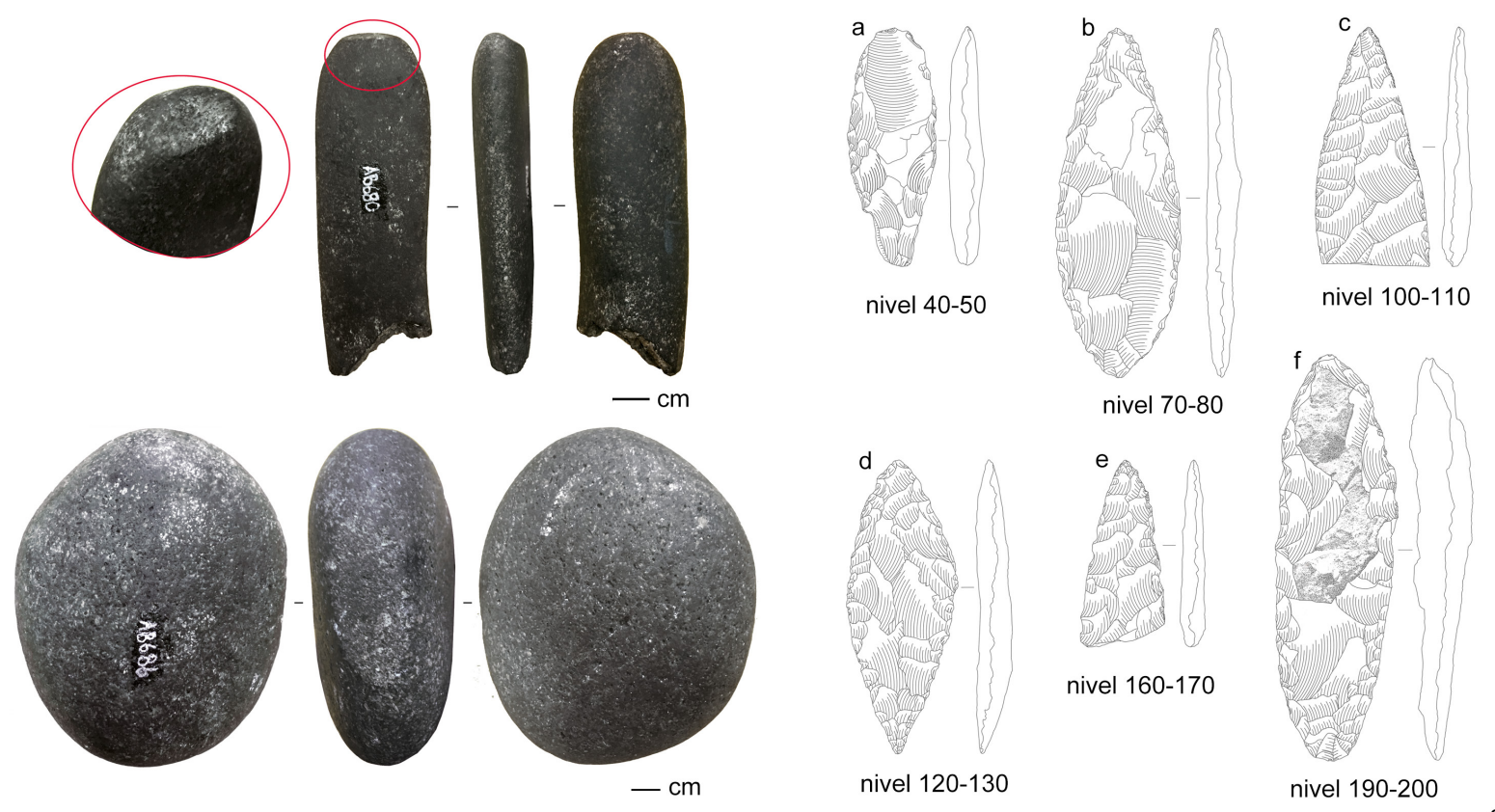

Fig. 3. Material lítico recuperado en San Juan 1. Izq.: Instrumentos de piedra piqueteada y abradida. Der.: Dibujos de bifaces obtenidos en diferentes niveles de excavación.

de guijarros con un notable aplanamiento en una o dos secciones de sus bordes, en algunos casos, con ligeras marcas de abrasión y estrías (Fig. 3).

Una característica de suma relevancia del conjunto es el predominio de obsidiana riolítica procedente de la fuente de aprovisionamiento del volcán Chaitén, ubicada a $95 \mathrm{~km}$ al sureste del sitio, en Chiloé Continental, abarcando el $81 \%$ de la muestra. También se registra un $19 \%$ de otras rocas como basalto y riolita, entre otras materias primas. Estas son de buena a muy buena calidad para la talla concoidal. La obsidiana riolítica gris fue utilizada especialmente en la confección de instrumentos y bifaces, al igual que lo documentado para otros sitios arqueológicos del archipiélago septentrional (Stern et al. 2002; Munita, 2007; Reyes et al. 2015). La obsidiana de Chaitén tuvo amplia circulación en Patagonia occidental (Stern, 2018) y nos refiere a los amplios rangos de circulación e intercambio por parte de los grupos canoeros a lo largo de toda la secuencia, desde el Holoceno medio hasta el Holoceno tardío (Reyes et al. 2016, 2019a).

En cuanto a la subsistencia, los estudios arqueobotánicos, específicamente carporrestos y microfósiles, revelan el uso y consumo de plantas silvestres nativas (González, 2019). Respecto al registro arqueofaunístico ( $\mathrm{N}=4.911)$, la explotación de mamíferos está dominada por otáridos, seguida de Pudú (Pudu puda). También se registra consumo de delfines y coipos. Los restos de aves están escasamente representados, siendo predominantes los restos de aves marinas como pingüinos y cormoranes (San Román, 2019). La tecnología ósea fue registrada muy discretamente; un fragmento de punta ósea pequeña y una diáfisis de costilla de ballena con huellas de corte perimetral además de un hueso de cánido perforado. Los peces, en tanto, jugaron un rol central en la subsistencia. Las especies más frecuentes corresponden a jurel (Thrachurus murphyi) y pejerrey (Odontesthes sp.), destacando una alta riqueza taxonómica (12 especies) asociada a ambientes de captura diversos (Torres, 2019). Esto permite plantear el empleo de técnicas de captura de orilla y a través del uso de embarcaciones, probablemente ambas asociadas al uso de líneas como lo apoya la presencia de pesas de red. La explotación de recursos malacológicos, por otro lado, se registra homogénea (12 taxa) relacionada con una importante recolección de fauna de fondo arenoso, rocoso y mixto obtenida de la costa adyacente. Principalmente se registran bivalvos como las almejas (Venus antiqua y Semele solida) (Cárcamo, 2018). 
En relación a los sitios Tauco 1 y 2 , se configura un panorama distinto respecto a la ocupación del área. La cronología base de Tauco 1 nos habla del inicio de la ocupación del lugar en torno a los $\sim 3.000$ años cal AP con mayor intensidad de ocupación en torno a los 2.000 años cal AP, en relación a la cantidad de material fechado relacionado con actividad humana (sensu Rick, 1987), y donde se registra una alta tasa de acumulación de conchas desde la base del área más cercana a la costa hasta los niveles superiores (Tabla 1). Hasta los $80 \mathrm{~cm}$ de profundidad, también se registra un alto grado de disturbación antrópica subactual, en cuyos niveles se recuperaron fragmentos cerámicos monocromos $(\mathrm{N}=4)$, asignables al Período Alfarero tardío (Palma \& Alfaro, 2019), mezclados con basura subactual.

En cada una de las intervenciones realizadas en Tauco 1 se recuperaron restos óseos fragmentados e incompletos atribuidos a inhumaciones individuales. Se recuperaron los restos pertenecientes a 5 individuos (Fig. 2): un adolescente de sexo indeterminado entre 16-20 años (Ind. 1); un adulto joven, masculino, entre 28-36 años (Ind. 3); un niño de sexo indeterminado entre 0 a 7 años (Ind. 4); un adulto medio, femenino, entre 40-50 años (Ind.5) $y$ un infante de sexo indeterminado entre 2-10 años identificado en laboratorio (Ind.6) (Urrutia, 2019). En Tauco 2, en tanto, se recuperaron los restos de un individuo adulto medio, entre 25-45 años de sexo indeterminado (Ind. 1) en la base del conchal. Los individuos inhumados en ambos sitios, salvo el individuo 3 de Tauco 1 , se concentran temporalmente, al igual que el set de fechados de carbones, en torno a la intensidad de ocupación de los 2.000 años cal AP (Tabla 1). Todos los individuos presentan valores de isótopos obtenidos del colágeno de huesos de $\delta^{13} \mathrm{C}$ (entre -12.27 y -11.51) y $\delta^{15} \mathrm{~N}$ (entre 17.79 y 19.97) presentando una dieta eminentemente marina (Reyes et al. 2019b). Por otro lado, el patrón inhumatorio, decúbito lateral flectado (derecho) registrado en el individuo 5 de Tauco 1 (Fig. 4) e individuo 1 de Tauco 2, también es descrito a lo largo del archipiélago septentrional (Díaz \& Garretón, 1972/73; Rivas et al. 1999; Munita et al. 2011; Reyes, 2017).

Más allá de la serie de inhumaciones individuales registradas, las excavaciones muestran una serie de eventos diferenciados de descarte de

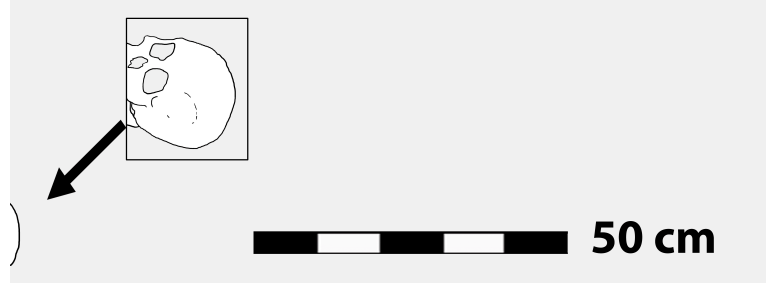

Fig. 4. Dibujo planta Unidad 2 del sitio Tauco 1. Registro inhumación Individuo 4 (TAU1-4, cráneo en perfil) e Individuo 5 (TAU1-5, completo). Dibujo gentileza de Ricardo Álvarez.Abel. 
material malacológico (principalmente bivalvos y Mytilus sp.), fogones y lentes de ceniza, no obstante, la cantidad de material cultural recuperado es muy baja respecto al volumen de sedimentos removidos: 4 fragmentos cerámicos, 10 desechos de talla, tres guijarros con percusión y un yunque. Es posible que otras áreas de actividades con mayor descarte de materiales se encuentren aún sin registrar alrededor, bajo los grandes volúmenes de sedimento conchífero acumulado (sensu Stein et al. 2003), o bien hayan sido destruidas por la erosión que las mareas provocan en el frente costero del sitio.

\section{DISCUSIÓN Y CONCLUSIONES}

De acuerdo a la información ecofactual y artefactual recuperada podemos señalar que el sitio San Juan 1 corresponde a un extenso $y$ potente conchal formado por reiteradas ocupaciones por parte de grupos de cazadores recolectores pescadores que visitaron el lugar desde hace $~ 6.000$ años cal AP, y en el que realizaron múltiples actividades de campamento en torno a prácticas de subsistencia orientadas a la recolección de mariscos y plantas, la pesca y la caza de aves marinas, y de mamíferos terrestres y marinos. Su secuencia de ocupación se prolonga hasta el Holoceno tardio, incluyendo la presencia de tecnología cerámica prehispánica tardía en los niveles superiores. Al igual que lo documentado en sitios del Holoceno medio en Chiloé como Puente Quilo 1, Chepu 005 y Yaldad 2 (Rivas et al. 1999; Legoupil, 2005; Álvarez et al. 2008; Rivas \& Ocampo, 2010), los componentes tecnológicos, especialmente las puntas lanceoladas de sección espesa, la utilización de materias primas líticas de amplia circulación (obsidiana riolítica), junto con un patrón de asentamiento eminentemente costero a lo largo de su secuencia, incluyendo niveles alfareros, y las características de los recursos explotados, nos refieren a la similitud contextual de los grupos canoeros que ocuparon inicialmente la isla, en donde además se observa que las mismas locaciones son permanentemente frecuentadas, independientemente de los cambios del nivel del mar, a lo largo de la secuencia. Esta situación es contrastante respecto a las ocupaciones iniciales del resto de los canales septentrionales (archipiélago de los Chonos), donde no hubo reutilización de los lugares inicialmente visitados, con cambios geomorfológicos de por medio y con conchales documentados recién hacia los $\sim 3.500$ años cal AP (Reyes et al. 2018, 2019a).

En los sitios Tauco 1 y 2 , registramos ocupaciones a partir del Holoceno tardío ( 3.000 años cal AP), en un área ya ocupada desde el Holoceno medio con formación de grandes montículos conchales como el de Rauco (Álvarez et al. 2008), por grupos que practicaron un modo de subsistencia especializado en la caza y recolección fundamentado en recursos del medio litoral y marino, como hemos visto ya de larga data en el área, y tal como lo refrendan los valores de isótopos estables de los 5 individuos fechados y en sintonía con los valores determinados para los cazadores recolectores marinos de Patagonia occidental (Reyes et al. 2019c). Estos sitios, de acuerdo a las dataciones obtenidas $(\mathrm{N}=9)$, nos remiten a una mayor intensidad de ocupación del lugar hacia los 2.000 años cal AP, y a la continuidad invariable de una tradición de cazadores recolectores marinos. También observamos en ellos un componente cerámico Alfarero Tardío, bajamente representado en las capas superiores, y como regla general, muy alterado por las disturbaciones antrópicas históricas y subactuales.

Los sitios descritos reflejan secuencias y momentos distintos de ocupación en el mar interior de Chiloé. La contextualización de 15 nuevos fechados radiocarbónicos aporta a una base de datos en permanente construcción sobre los yacimientos arqueológicos de la isla, formada principalmente por manuscritos e informes de acceso discrecional y que, ciertamente, contribuye con elementos de juicio para caracterizar el modo de vida cazador recolector marino del archipiélago septentrional.

\section{AGRADECIMIENTOS}

Financiamiento FONDECYT №1170726. A Doris Barría y Armando Bahamonde, pobladores y amigos de San Juan. A las hermanas Carolina y Consuelo Bahamonde de San Juan, y Francisco Aude de Tauco, por las facilidades y ayuda prestada para trabajar en su propiedad. A Felipe Montiel, Director del Museo Municipal de Castro, 
por su colaboración con nuestro Proyecto. A los antropólogos David Núñez, por sus gestiones y ayuda para acceder a la investigación en Tauco, y Ricardo Álvarez, por su colaboración en terreno y sus dibujos. Al equipo de arqueólogos: Francisco Cayla, Ximena Albornoz, Pablo González y Karol González. Al equipo de analistas: Jimena Torres, Javier Cárcamo, Silvia Alfaro, Gabriela Palma, Constanza Arecheta y Constanza Urrutia.

\section{BIBLIOGRAFÍA}

Aldunate, C. (1996). Mapuche: gente de la tierra. En J. Hidalgo, V. Schiappacasse, H. Niemyer, C. Aldunate \& P. Meges (Eds), Nueva historia de Chile desde los orígenes hasta nuestros días (pp.111-134). Santiago de Chile: Editorial Universitaria.

Álvarez, R., Munita, D., Fredes, J., \& Mera, R. (2008). Corrales de pesca en Chiloé. Valdiia: Imprenta América.

Álvarez, R., Munita, D., Mera, R., Borlando, I., Ther, F., Núñez, D., Hidalgo C., \& Hayward, P. (2019). Rebounding from extractivism: The history and re-assertion of traditional weir-fishing practices in the Interior Sea of Chiloé. Shima, 13(2), 155-173.

Aspillaga, E., Ocampo, C., Olivares, J., Arensburg, B., \& Meyer, J. (1995). Una visita a los canoeros de Quetalmahue. Revista Museos, 20, 18-20.

Bibar, G. (1979) [1558]. Crónica y relación copiosa y verdadera de los Reinos de Chile, Berlín: Edición de L. Sáez-Godoy, Bibliotheca Ibero-Americana, ColloquiumVerlag.

Cárcamo, J. (2018). Análisis arqueomalacológico sitio San Juan 1, Archipiélago de Chiloé, Región de Los Lagos. (PROYECTO FONDECYT N 1170726). Ms.

Coltrain, J., Tackney, J., \& O־Rourke, D. (2016). Thule Whaling at Point Barrow, Alaska: The Nuvuk Cemetery Stable Isotope and Radiocarbon Record. Journal of Archaeological Science, Reports, 9, 681-694.

Cooper, J. (1917). Analytical and critical bibliography of the tribes of Tierra del Fuego and adjacent territory. Washington: Smithsonian Institution.

Cooper, J. (1946). The Chono. En J. Steward (Ed.) Handbook of South American Indians. Vol. 1: The Marginal Tribes (pp. 47-54). Bureau of American Ethnology Bulletin. (143). Washington DC: Smithsonian Institution.

De Ercilla, A. (2009) [1569]. La Araucana. Madrid: Edición de Isaías Lerner, Cátedra.

Díaz, C., \& Garretón, M. (1972/73). El poblamiento prehispánico del área insular septentrional chilena. En
Actas del VI Congreso de Arqueología chilena (559584).

Gaete, N., Navarro, X., Constantinescu, F., Mera, R., Selles, D., Solari, M., Vargas, L.,... Durán, L. (2004). Una mirada al modo de vida canoero del mar interior desde Piedra Azul. Chungara Revista de Antropología, Volumen especial I, 333-346.

Goicueta, M. (1558). Derrotero y viaje de Juan Ladrillero. Sevilla: Archivo General de Indias, Fondo Patronato, 32, R. 5-1, fjs. $1-44 v$.

González, K. (2019). Uso de recursos vegetales por grupos cazadores-recolectores maritimos en el sitio San Juan 1, Chiloé (6.000-2.000 años cal. AP). Memoria título profesional. Escuela de Arqueología, Departamento de Antropología, Facultad de Ciencias Sociales, Universidad Alberto Hurtado. Ms.

Hogg, A., Hua, Q., \& Blackwell, P. (2013). SHCAL13 Southern hemisphere calibration, 0-50,000 years CAL BP. Radiocarbon, 55 (4), 1889-1903.

Legoupil, D. (2005). Recolectores de mariscos tempranos en el sureste de la isla de Chiloé. Magallania, 33(1), 51-61.

Legoupil, D., \& Fontugne, M. (1997). El poblamiento marítimo en los archipiélagos de Patagonia: núcleos antiguos y dispersión reciente. Anales del Instituto de la Patagonia, Serie Ciencias Humanas, 25, 75-87.

Luebert, F., \& Pliscoff, P. (2006). Sinopsis bioclimática y vegetacional de Chile. Santiago de Chile: Editorial Universitaria.

Menghin, O. (1962). Estudios de prehistoria araucana. Studia Praehistorica2. Buenos Aires: Centro Argentino de Estudios Prehistóricos.

Merino V., De Pol-Holz, R., Southon, J., Latorre, C., \& Collado, S. (2019). Marine radiocarbon reservoir age along the chilean continental margin. Radiocarbon, 61(1), 195-210.

Morello, F., \& Arecheta, C. (2019). Informe material litico sitio San Juan 1, Comuna de Dalcahue, Chiloé. (PROYECTO FONDECYT N 1170726). Ms.

Munita, D. (2007). Materias primas líticas en sitios costeros del extremo sur septentrional de chile. Dispersión y aprovisionamiento. Boletín de la Sociedad Chilena de Arqueología, 39, 103-112.

Munita, D., Mera, C. R., Arregui, I., \& Manneschi, M. (2011). Funebria de grupos canoeros durante el Holoceno tardío en la región de los lagos. El conchal de Yaco Alto1, Calbuco, Chile. ARGENTINA: Revista cazadores recolectores del cono sur, 6, 17-41.

Ocampo, C., \& Rivas, P. (2004). Poblamiento temprano de los extremos geográficos de los canales patagónicos: Chiloé e isla Navarino 1. Chungara. Volumen 
Especial, 1, 317-331.

Orquera, L., \& Piana, E. (2006). El poblamiento inicial del área litoral sudamericana sudoccidental. Magallania, 34(2), 21-36.

Orquera, L., Legoupil, D., \& Piana, E. (2011). Littoral adaptation at the southern end of South America. Quaternary International 239, 61-69.

Palma, G., \& Alfaro S. (2019). Informe de análisis cerámica de sitios arqueológicos de la Provincia de Chiloé, Región de los Lagos. (PROYECTO FONDECYT $\left.\mathrm{N}^{\circ} 1170726\right)$.

Reimer, P. J., Bard, E., Bayliss, A., Beck, W. J., Blackwell, P., Ramsey, C., Buck, C.E.,... \& Van Der Plicht J. (2013). IntCal13 and Marine13 Radiocarbon Age Calibration Curves, 0-50,000 years cal BP. Radiocarbon, 55, 1869-1887.

Reyes, O. (2017). El poblamiento del archipiélago de los Chonos $\left(43^{\circ}-47^{\circ}\right.$ S). Patagonia occidental. Chile. Tesis doctoral. Facultad de Filosofía y Letras, Universidad de Buenos Aires. Ms.

Reyes, O., Méndez, C., San Román, M., \& Francois, J. (2018). Earthquakes and coastal archaeology: Assessing shoreline shifts on the southernmost Pacific coast (Chonos Archipelago 4350"-4650" S, Chile, South America). Quaternary International, 463, 161-175.

Reyes, O., Méndez, C., \& San Román, M. (2019a). Cronología de la ocupación humana en los canales septentrionales de Patagonia occidental, Chile. Intersecciones en Antropología, 20(2), 153-165.

Reyes, O., Moraga, M., Méndez, C., \& Cherkinsky, A. (2015). Maritime Hunter-Gatherers in the Chonos Archipelago

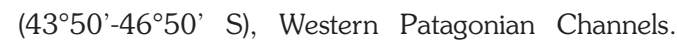
Journal of Island and Coastal Archaeology, 10(2), 207-231.

Reyes, O., San Román, M., \& Morello, F. (2016). Search for maritime hunter-gatherer archaeological record in the shifting shorelines of the South Pacific Coast (Chonos and Guaitecas Archipelago, Chile). En H. Bjerck, H. Breivik, S. Fretheim, E. Piana, B. Skar, A. Tivoli \& F. Zangrando (Eds.), Marine Ventures: Archaeological Perspectives on Human-Sea Relations (pp. 141-155). UK: Equinox Publishing.

Reyes, O., Tessone, A., Belmar, C., San Román, M., Morello, F., Urbina, X., \& Moraga, M. (2019b). Trayectorias culturales y subsistencia en el Archipiélago de Chiloé, Patagonia, Chile: Cambios e interacción entre sociedades cazadoras-recolectoras y agro-alfareras en áreas insulares durante el Holoceno tardío. Poster. III Taller de Arqueología e Isótopos en el Sur de
Sudamérica. 24-27 Septiembre, Pica: CHILE.

Reyes, O., Tessonne, A., San Román, M. \& Méndez, C. (2019c). Dieta e isótopos estables de cazadores recolectores marinos en los canales occidentales de Patagonia, Chile. Latin American Antiquity, 30(3), 550-578.

Rick, J. (1987). Dates as Data: An examination of the Peruvian Preceramic radiocarbon record. American Antiquity, 52, 55-73

Rivas, P., \& Ocampo, C. (2010). La adaptación humana al bosque en la isla de Chiloé. Estrategias adaptativas en el litoral septentrional de los canales patagónicos. Valdivia: Actas del XVII Congreso Nacional de Arqueología Chilena (2006). T2, 1449-1460.

Rivas, P., Ocampo, C., \& Aspillaga, E. (1999). Poblamiento Temprano de los Canales Patagónicos: El Núcleo Ecotonal Septentrional. Anales del Instituto de la Patagonia, 27, 221- 230.

San Román, M. (2014). Sea-level changes and coastal peopling in southernmost Pacific South America: Marine hunters from Patagonia. Springer New York: Encyclopedia of Global Archaeology, 6515-6525.

San Román, M. (2019). Análisis arqueofaunístico sitio San Juan 1, Comuna de Dalcahue, Chiloé. (PROYECTO FONDECYT No 1170726). Ms.

SHOA (2019). Tablas de Marea de la Costa de Chile. Valparaíso: Ediciones Servicio Hidrográfico y Oceanográfico de la Armada de Chile.

Sierralta, S., Delgado, A., Kelly, P., \& Rebolledo, S. (2019). Cronología absoluta en los canales septentrionales, el mar interior y la costa pacífica austral. Arqueología de la Patagonia: El pasado en las arenas. Actas de las X Jornadas de Arqueología de la Patagonia. (pp.167179). Puerto Madryn: ARGENTINA.

Stein, J., Deo, J., \& Phillips, L. (2003). Big sites-short time: accumulation rates in archaeological sites. Journal of Archaeological Science, 30(3), 297-316.

Stern, C. (2018). Obsidian sources and distribution in Patagonia, southernmost South America. Quaternary International, 468, 190-205

Stern, C., \& Porter, C. (1991). Obsidiana en yacimientos arqueológicos de Chiloé y las islas Guaitecas. Anales del Instituto de la Patagonia. Serie Ciencias Humanas, 20, 205-209.

Stern, C., Navarro, X., \& Muñoz, J. (2002). Obsidiana gris translúcida del volcán Chaitén en los sitios arqueológicos de Quilo (Isla Grande de Chiloé) y Chanchán (X Región) Chile, y obsidiana del Mioceno en Chiloé. Anales del Instituto de la Patagonia, Serie Ciencias Humanas, 
30, 167-174

Stuiver, M., Reimer, P.. \& Reimer, R. (2013). Recuperado de CALIB 7.0.2 www program and documentation. http://calib.qub.ac.uk/calib/.

Torres, J. (2019). Análisis ictioarqueológico sitio San Juan 1, Comuna de Dalcahue, Chiloé. (PROYECTO FONDECYT N $\left.{ }^{\circ} 1170726\right)$. Ms.

Urbina, X., Reyes, O., \& Belmar, C. (2020). Canoeros en Chiloé: de facilitadores de las navegaciones españolas en los archipiélagos de los Chonos y de Guayaneco, a productores y comerciantes. Chungara, Revista de Antropología, 1567-1792. http://dx.doi.
org/10.4067/S0717-73562020005000702.

Urrutia, C. (2019). Informe de análisis bioantropológico: Sitios Tauco 1 y Tauco 2. (PROYECTO FONDECYT $\left.\mathrm{N}^{\circ} 1170726\right)$. Ms.

Vásquez de Acuña, I. (1963). Arqueología Chiloense: Yacimientos y Material Lítico. En Trabajos de Prehistoria 12:5. Madrid: Instituto Español de Prehistoria del Consejo Superior de Investigaciones Científicas.

Zangrando, F. (2018). Poblamiento temprano y arqueología de costas en Patagonia y Tierra del Fuego: vacío de información, preconceptos y perspectivas. Intersecciones en Antropología, 19(2), 63-97. 